\title{
Milk Fat Globule Membrane Supplementation Promotes Neonatal Growth and Alleviates Inflammation in Low-Birth-Weight Mice Treated with Lipopolysaccharide
}

\author{
Shimeng Huang, ${ }^{1}$ Zhenhua Wu, ${ }^{1}$ Cong Liu, ${ }^{1}$ Dandan Han, ${ }^{1}$ Cuiping Feng, \\ Shilan Wang, ${ }^{1}$ and Junjun Wang $\mathbb{D}^{1}$ \\ ${ }^{1}$ State Key Laboratory of Animal Nutrition, College of Animal Science and Technology, China Agricultural University, \\ Beijing 100193, China \\ ${ }^{2}$ Department of Obstetrics and Gynecology, China-Japan Friendship Hospital, Beijing 100029, China
}

Correspondence should be addressed to Junjun Wang; wangjj@cau.edu.cn

Received 24 February 2019; Accepted 15 April 2019; Published 2 May 2019

Guest Editor: Deguang Song

Copyright $\odot 2019$ Shimeng Huang et al. This is an open access article distributed under the Creative Commons Attribution License, which permits unrestricted use, distribution, and reproduction in any medium, provided the original work is properly cited.

\begin{abstract}
Impaired intestinal mucosal integrity and immunity are frequently observed in low-birth-weight (LBW) animals, which lead to inadequate growth and high neonatal mortality. However, the mechanisms of intestinal dysfunction in LBW animals are still unclear. Milk fat globule membrane (MFGM), a protein-lipid complex surrounding the fat globules in milk, has many healthful benefits for animals. Therefore, this study was conducted to explore the effect of MFGM supplementation on intestinal injury and inflammation in LBW mouse pups while being challenged with lipopolysaccharide (LPS). C57BL/6J LBW female neonatal mice were fed on breast milk and divided into four groups, including two normal diet groups (ND; CON group and LPS group) and the diet supplemented with two dosages of MFGM, namely, MFGM100 (ND plus MFGM at $100 \mathrm{mg} / \mathrm{kg}$ BW) and MFGM200 (ND plus MFGM at $200 \mathrm{mg} / \mathrm{kg} \mathrm{BW}$ ) from postnatal day (PND) 4 to PND 21. At PND21, pups from the LPS group, MFGM100 group, and MFGM200 group were injected intraperitoneally with LPS while the pups from the CON group were injected with equivalent volume of sterile saline. After $4 \mathrm{~h}$ of LPS administration, all pups were slaughtered and then the plasma, mid-ileum, and mid-colon tissue samples were collected. Our results showed that MFGM supplementation promoted the body weight from PND16 to PND21 and attenuated intestinal inflammation manifested by reduced histological damage, decreased secretion of TNF- $\alpha$, IL- 6 , IFN- $\gamma$, and IL-1 $\beta$, and improved oxidative stress characterized by increased SOD activity and decreased secretion of MDA. Expression of tight junction proteins (ZO-1, occludin, and claudin-1), MUC1, and MUC2 was increased in MFGM presupplemented groups compared to the LPS-challenged mice with normal diet. Meanwhile, the expression of proinflammatory cytokines and TLRs was decreased by MFGM presupplementation. Collectively, MFGM is a critical nutrient with an ability to improve the growth performance of LBW mouse pups, especially during the LPS challenge, by promoting the intestinal epithelial integrity and inhibiting inflammation through activating of TLR2 and TLR4 signals.
\end{abstract}

\section{Introduction}

Infants with low birth weight (LBW) have higher morbidity and mortality than normal birth weight infants during their neonatal period as an outcome of intrauterine growth restriction (IUGR) [1], which was defined as impaired growth and development of the mammalian embryo/fetus or its organs during pregnancy [2]. Despite advanced prenatal care for both mothers and fetuses, approximately $15 \%$ of human infants suffer from LBW worldwide [3] and approximately $15-20 \%$ of newborn piglets suffer from IUGR [4]. Neonates with LBW show impaired intestinal development, nutrient metabolism, and immune function in both human-beings $[5,6]$ and animal models $[2,7,8]$. Metabolic programming and the alterations in early physiological may proceed to intestinal dysfunction during the neonatal period [9]. During this critical window, nutrition is the principal contributor to the development of immune and metabolic and the 
establishment of microbes [10]. Epidemiological studies also indicated that the LBW humans or animals are in high risk of metabolic disease in their later life [11]. Therefore, exploring novel strategies to improve the development of LBW animals or human infants during their suckling period is paramount and has prospects for practical applications.

Milk fat globule membrane (MFGM), derived from the apical surface of mammary epithelial cells and composed of proteins and lipids [12], could protect the milk fat globules and retard their physical destabilization in milk $[13,14]$. Several studies have found that supplementation of the MFGM could decrease the infection and inflammation in rodent models [15] and promote the gut mucosal integrity during lipopolysaccharide (LPS)-induced intestine inflammation in male BALB/c adult mice [16], suggesting that MFGM possess a function of antibacterial and anti-inflammatory activity [16]. In addition, MFGM has attracting attentions in related metabolic disorders in high fat diet induced obese mice [17]. MFGM treatment on human infants and neonatal piglets could improve the neurodevelopment and increase the cognitive as compared to the control formula, reaching a similar effect to those of breastfed infants [18-20]. However, previous studies showed no significant effects of MFGM supplementation on weight gain and feed intake of normal birth weight mice during early life and adulthood time $[16,21]$. Whether MFGM can promote the growth of LBW newborns or alleviate the intestinal inflammation and injury induced by LPS challenge is unknown. We hypothesized that MFGM supplementation could attenuate the intestinal inflammation and damage in LBW pups, especially while being challenged with LPS. Therefore, the present study was conducted to investigate the protective effect of MFGM against intestinal injury in LBW mice during neonatal growth and LPS challenge, as well as the possible mechanisms.

\section{Materials and Methods}

2.1. Animals and Treatments. All experiments were performed in accordance with the Health Guide for Care and Use of Laboratory Animals by China Agricultural University. C57BL/6J female mice were obtained from Sibeifu Inc. (Beijing, China). All mice were housed under a $12 \mathrm{~h}$ light/dark cycle, a constant temperature $\left(24^{\circ} \mathrm{C}\right)$, and $50 \%$ humidity. Pregnant mice were housed individually with ad libitum access to normal chow diet (KeAoXieLi Feed Co., Ltd., Beijing, China). At birth, pups were spontaneously delivered from female mice and their body weights were recorded. A low-birth-weight (LBW) pup was defined when its birth weight was lower than 2 SD (standard deviations) of the mean birth weight of their littermates [22, 23]. On postnatal day (PND) 4, LBW female mice $(\mathrm{n}=32)$ were cross-fostered to adjust the litter size among these groups and were divided into four groups ( $\mathrm{n}=8$ pups/group), including two normal diet groups (ND; CON group and LPS group) and the diet supplemented with two dosages of MFGM, namely, MFGM100 (ND plus MFGM at $100 \mathrm{mg} / \mathrm{kg}$ BW) and MFGM200 (ND plus MFGM at $200 \mathrm{mg} / \mathrm{kg} \mathrm{BW)} \mathrm{from}$ PND4 to PND 21. Meanwhile, body weight was measured every day and dosage of MFGM administration was adjusted.
From PND4 to PND11, pups were administered in a volume averaged $20 \mu \mathrm{L}$ and $50 \mu \mathrm{L}$ from PND11 to PND21. At PND21, pups from the LPS group, MFGM100 group and MFGM200 group were injected intraperitoneally with LPS $(10 \mathrm{mg} / \mathrm{kg}$ BW; E. coli serotype 055: B5, Sigma Chemical) while the pups from the CON group were injected with equivalent amount of sterile saline.

2.2. Tissue Sampling. After $4 \mathrm{~h}$ of LPS treatment, all the pups were anesthetized with tribromoethanol $(500 \mathrm{mg} / \mathrm{kg})$ and blood was taken to collect plasma and the mid-ileum and mid-colon were dissected. The blood was centrifuged for 15 minutes at $3000 \mathrm{rpm}$ and plasma stored at $-20^{\circ} \mathrm{C}$. Intestinal tissues (mid-ileum and mid-colon) were harvested immediately and fixed in $4 \%$ buffered formalin overnight and processed for routine histological analysis. Meanwhile, the mid-ileum and mid-colon were washed with normal saline, snapped frozen in liquid nitrogen, and stored at $-80^{\circ} \mathrm{C}$ prior to processing for ELISA and qRT-PCR analysis of the cytokines.

2.3. Histological Analysis. The paraformaldehyde-fixed midileum and mid-colon were dehydrated in graded alcohol and embedded in paraffin wax. Then, hematoxylin and eosin (H\&E) stained paraffin sections were viewed under bright field on a Zeiss Axio Imager microscope as outlined previously. Microscopic intestinal damage was observed in images using the measurement tool on CaseViewer software at 200x magnification. The degree of intestinal tissue damage was scored as described by Ji et al. [24] and Nishiyama et al. [25], where the extent of epithelial loss on intestinal villi and inflammatory infiltration was evaluated and included in the histopathological examination.

2.4. Plasma Inflammatory Profile Analysis. The concentrations of proinflammatory cytokines (TNF- $\alpha$, IL- 6 , and IL- $1 \beta$ ) were detected using enzyme-linked immunosorbent assay (ELISA) kits according to the protocol provided (eBioscience, CA, USA). Meanwhile, the concentrations of malonyldialdehyde (MDA) and superoxide dismutase (SOD) were analyzed by kits from Nanjing Jiancheng Bioengineering Institute (Nanjing, China).

2.5. RNA Extraction and Quantitative Real-Time PCR Analysis. Total RNA from mid-ileum and mid-colon were extracted using TRIzol kit (Invitrogen, Carlsbad, CA, USA) following the protocol. cDNA was obtained using PrimeScript $^{\mathrm{TM}}$ RT Kit (Takara, Japan). The qPCR was performed according to the SYBR Premix $\mathrm{Ex}^{\mathrm{Taq}^{\mathrm{TM}}}$ II instructions (Takara, Japan). The reaction was performed on a LightCycler $^{\circledR}$ System (Roche, Germany). Primers for RT-qPCR were synthesized by Shanghai Generay Biotech Co., Ltd. (Supplementary Table S1). Amplifications were performed in triplicate for each sample. The relative abundances of target genes to that of the reference gene ( $\beta$-actin) were calculated according to $2^{-\Delta \Delta \mathrm{Ct}}$ method.

2.6. Statistical Analysis. The statistical significance of differences among means was assessed with one-way analyses of variance (ANOVA) and Student-Newman-Keuls test. All the 
TABLE 1: Effect of MFGM supplementation from postnatal d 4 to d 21 on plasma proinflammatory cytokines, antioxidant enzyme activity, and oxidant products in LPS-challenged LBW mice ${ }^{\mathrm{a}}$.

\begin{tabular}{lcccc}
\hline Items & CON & LPS & MFGM100 & MFGM200 \\
\hline TNF- $\alpha(\mathrm{pg} / \mathrm{ml})$ & $19.14 \pm 1.80 \mathrm{a}$ & $37.02 \pm 4.84 \mathrm{c}$ & $27.84 \pm 3.16 \mathrm{~b}$ & $24.63 \pm 2.51 \mathrm{~b}$ \\
IL-6 $(\mathrm{pg} / \mathrm{ml})$ & $227.03 \pm 15.68 \mathrm{a}$ & $325.00 \pm 10.70 \mathrm{~d}$ & $282.04 \pm 15.97 \mathrm{c}$ & $251.99 \pm 12.94 \mathrm{~b}$ \\
IL-1 $\beta(\mathrm{pg} / \mathrm{ml})$ & $15.56 \pm 4.12 \mathrm{a}$ & $26.01 \pm 3.49 \mathrm{c}$ & $20.14 \pm 3.94 \mathrm{~b}$ & $25.69 \pm 3.45 \mathrm{c}$ \\
T-SOD $(\mathrm{U} / \mathrm{ml})$ & $109.69 \pm 3.30 \mathrm{a}$ & $101.45 \pm 11.58 \mathrm{a}$ & $127.18 \pm 7.92 \mathrm{c}$ & $111.70 \pm 8.05 \mathrm{ab}$ \\
MDA $(\mathrm{nmol} / \mathrm{ml})$ & $5.09 \pm 0.83$ & $5.38 \pm 0.80$ & $5.06 \pm 0.83$ & $5.32 \pm 0.45$ \\
\hline
\end{tabular}

${ }^{a}$ Mean values with their standard errors of the mean (SEM) ( $n=8 /$ group). Within a row, means without a common letter (a, b, c, and d) differ $(p<0.05)$.

data are expressed as mean \pm standard deviations (SD). $P$ $<0.05$ was regarded as statistically significant. The Prism software (Graphpad, San Diego, CA, USA) was used for all statistical analyses.

\section{Results}

3.1. MFGM Supplementation during Neonatal Stage Improved the Growth Performance of $L B W$ Mice. As shown in Figure 1(a), there was no difference in the body weight of LBW mice among the CON, LPS, MFGM100 and MFGM200 groups at beginning of the experiment (PND4). From PND16 to PND21, the body weight of MFGM100 and MFGM200 groups became significantly higher $(P<0.05)$ than that of the PBS gavaged treatments, due to the higher average daily gain with MFGM treatment $(P<0.05)$ (Figure $1(b))$. Finally, at PND21, the body weight of pups from the MFGM100 and MFGM200 groups were higher $(P<0.05)$ (Figure $1(\mathrm{c}))$ than the pups from the normal diet groups (CON and LPS).

3.2. MFGM Presupplementation Prevented the Intestinal Inflammatory Alterations Induced by LPS Challenge in LBW Mice. As shown in Figures 2 and 3, LPS-challenged mice had increased infiltration of inflammatory cells in the mucosal layer of ileal and colonic tissue compared with the control pup $(\mathrm{CON})$, while these harmful effects of LPS were significantly mitigated $(P<0.05)$ in MFGM100 and MFGM200 groups (Figures 2 and 3), suggesting that MFGM supplementation during the neonatal stage alleviated the LPS-induced intestinal injury in LBW pups. Meanwhile, feeding of the 100 $\mathrm{mg} / \mathrm{kg}$ BW MFGM showed better effects than MFGM200 in mitigating $(P<0.05)$ the LPS-induced intestinal damage.

To gain an insight into the effects of MFGM on secretional level of inflammatory cytokines in LPS-induced LBW pups, we assessed the concentrations of cytokines including TNF$\alpha$, IL-6, and IL- $1 \beta$ in the plasma. As shown in Table 1 , pups challenged with LPS (LPS group) had higher levels of TNF- $\alpha$, IL- 6 , and IL- $1 \beta$ than the CON group $(P<$ $0.05)$, which was largely relieved $(P<0.05)$ by MFGM supplementation, suggesting an effect of MFGM (MFGM100 and MFGM200 group) in inhibiting the secretion of proinflammatory cytokines. Furthermore, the supplementation of $100 \mathrm{mg} / \mathrm{kg}$ BW MFGM resulted in significantly lower concentrations of the proinflammatory cytokines (IL- 6 and IL-1 $\beta)(P<0.05)$ in pups than the MFGM200 group.

Expression level of the proinflammatory genes such as TNF- $\alpha$, IL- 6 , IFN- $\gamma$, and IL- $1 \beta$ was provided in Figures 4 and 5. In the ileum (Figure 4(a)), the pups challenged with LPS had higher expression of TNF- $\alpha$, IL-6, IFN- $\gamma$, and IL- $1 \beta$ $(P<0.05)$, compared to the mice in CON group. However, the MFGM presupplementation (MFGM100 or MFGM200 group) relieved $(P<0.05)$ the LPS-induced intestinal damage by decreasing the gene expression levels of TNF- $\alpha$, IL- 6 , and IL-1 $\beta$. In the colon (Figure 5(a)), compared to the controls, the genes expressions of TNF- $\alpha$ and IL- $1 \beta$ were significantly increased $(P<0.05)$ in the LPS group, which were decreased $(P<0.05)$ by MFGM treatment (MFGM100 or MFGM200 group).

3.3. MFGM Presupplementation Prevented the Alterations of Plasma Antioxidant Index and Intestinal Antioxidant Gene Expressions Induced by LPS Challenge in LBW Mice. As shown in Table 1, the plasma activity of T-SOD was increased $(P<0.05)$ by MFGM presupplementation compared with the LPS group, and the MFGM100 had higher $(P<0.05)$ activity of T-SOD than MFGM200. LBW pups fed with $100 \mathrm{mg} / \mathrm{kg}$ BW MFGM (MFGM100 group) had also higher $(P<0.05)$ mRNA levels of CAT and SOD as compared with the controls in both ileum and colon, whereas there was no difference $(P>0.05)$ between the LPS group and CON group in ileum (Figures 4(c) and 5(c)). However, the expression level of SOD was lower $(P<0.05)$ in colon of the LPS group than those in other groups.

3.4. MFGM Presupplementation Regulated the Gene Expressions of Tight Junction and Inflammatory Pathway and Prevented Activation of the Toll-Like Receptors Induced by LPS Challenge in LBW Mice. To investigate whether the MFGM supplementation regulates intestinal tight junction, the gene expressions of ZO-1, claudin-1, and occludin in ileum and colon were measured. As shown in Figures 4 and 5, there were significant interactions $(P<0.05)$ between LPS challenge and intestinal tight junction or MUCs genes expression in ileum and colon. After the LBW pups were injected intraperitoneally with LPS for $4 \mathrm{~h}$, presupplementation of MFGM increased the mRNA expression levels of ZO-1, claudin-1, and occludin $(P<0.05)$ (Figures $4(\mathrm{~b})$ and $5(\mathrm{~b}))$. At the same time, the expression of MUC1 and MUC2 was significantly increased $(P<0.05)$ by MFGM administration (MFGM100 and MFGM200 groups) in both ileum and colon (Figures 4(b) and 5(b)), compared with the CON and LPS group.

Compared with the MFGM200 group, MFGM100 group had higher $(P<0.05)$ mRNA expression levels of ZO1, claudin-1, and occludin in both ileum and colon. After 


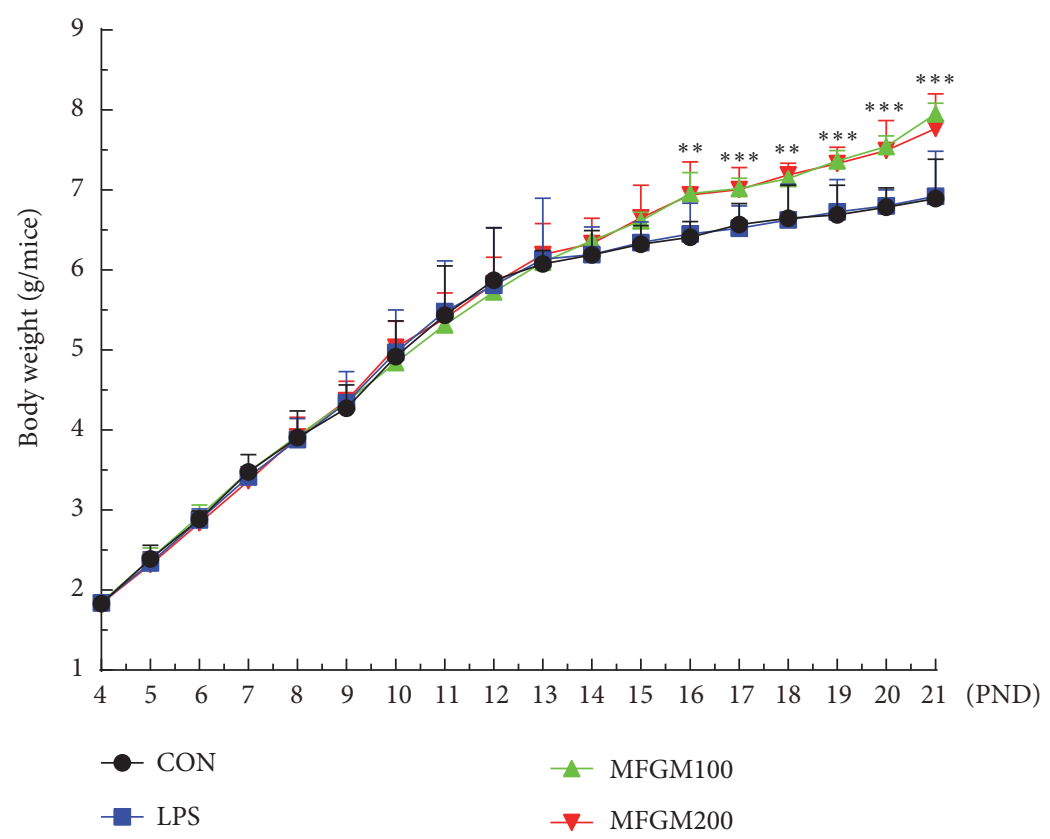

(a)

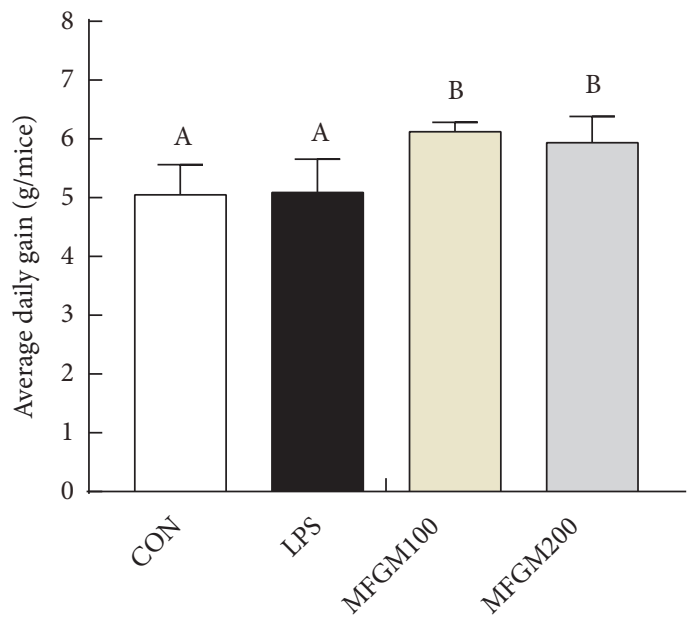

(b)

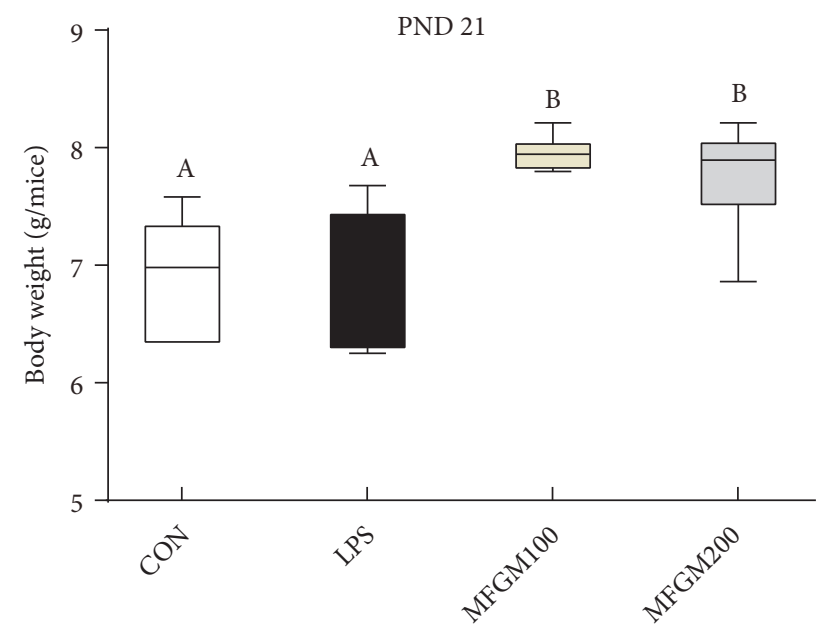

(c)

FIGURE 1: Effects of MFGM supplementation from postnatal d 4 to $d 21$ on the body weight gain in LBW mice. Before LPS being injected, pups were fed with phosphate buffered solution (CON and LPS group), MFGM at $100 \mathrm{mg} / \mathrm{kg} \mathrm{BW}$ (MFGM100), and 200 mg/kg BW (MFGM200) from PND4 to PND21. (a) Body weight from PND4 to PND21. (b) Average daily gain. (c) The body weight of pups at PND21. Mean values with their standard errors of the mean (SEM) ( $\mathrm{n}=8$ pups/group). Within a row, means without a common letter $(\mathrm{A}, \mathrm{B}$, and C) differ $(p<$ $0.05)$.

$4 \mathrm{~h}$ of LPS injection (Figures $4(\mathrm{~d})$ and $5(\mathrm{~d})$ ), the gene expressions of TLR2 and TLR4 in ileum and colon were higher $(P<0.05)$ in LPS group than those in the CON group, while were significantly decreased $(P<0.05)$ by MFGM presupplementation (MFGM100 and MFGM200 groups).

\section{Discussion}

The gastrointestinal tract (GIT) is of paramount importance in postnatal nutrient digestion and acquisition, where the epithelial barrier of the GIT plays an important function in immune system during neonatal period [26]. However, LBW or IUGR predispose the offspring to malnutrition and endanger the development of intestine and other organs after birth $[2,27,28]$. In current study, our results showed that MFGM supplementation from PND4 to PND21 improved the growth performance of LBW mice, and the MFGM presupplementation mitigated LPS-induced intestinal damage and inflammation at PND21. Our results suggested that this protective effect of MFGM is associated with reduced secretion of proinflammatory cytokines, increased antioxidant enzyme activity, and reduced gene expressions of TLR2 and TLR4. 

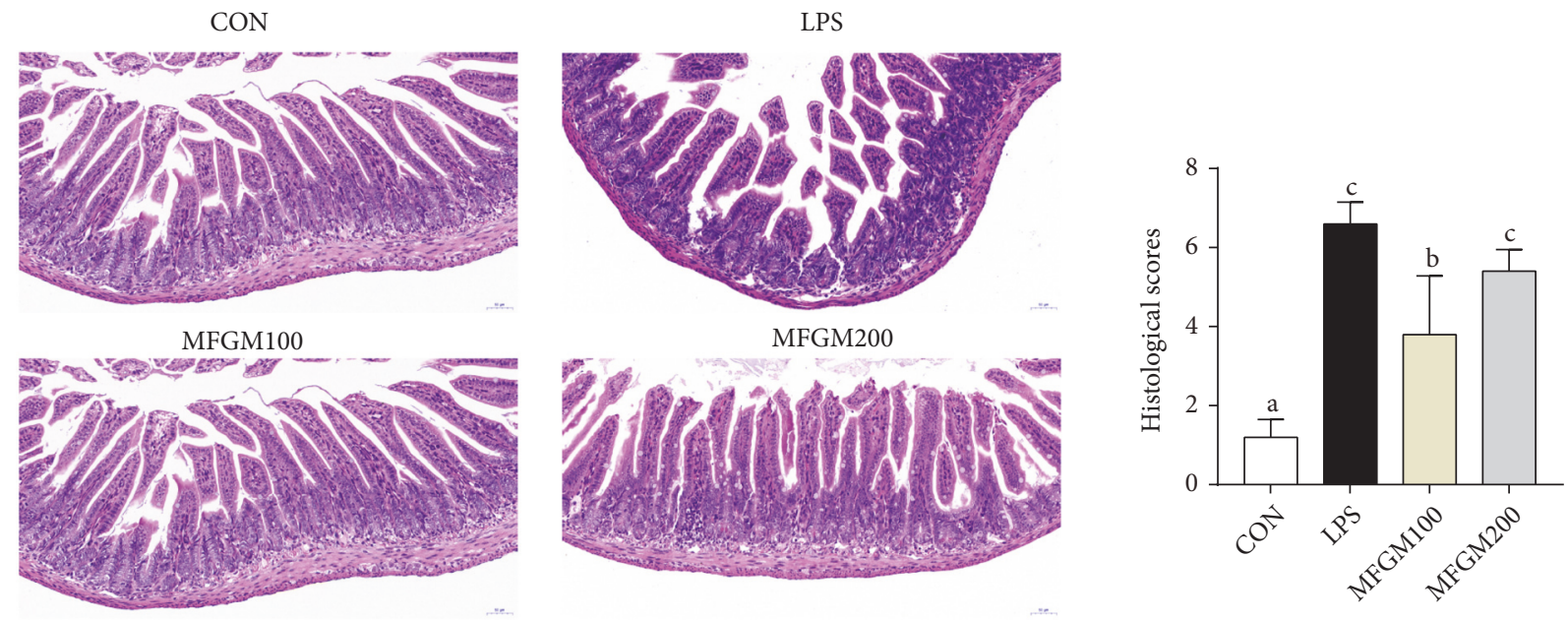

FIGURE 2: Effect of MFGM presupplementation from postnatal d 4 to d 21 on ileum damage in LBW mice after LPS challenge. Mean values with their standard errors of the mean (SEM) ( $\mathrm{n}=8$ pups/group). Within a row, means without a common letter $(\mathrm{a}, \mathrm{b}$, and $\mathrm{c})$ differ $(p<0.05)$.

CON

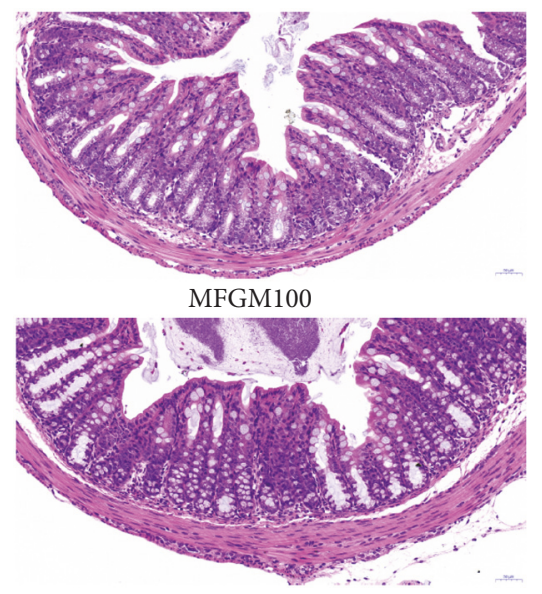

LPS

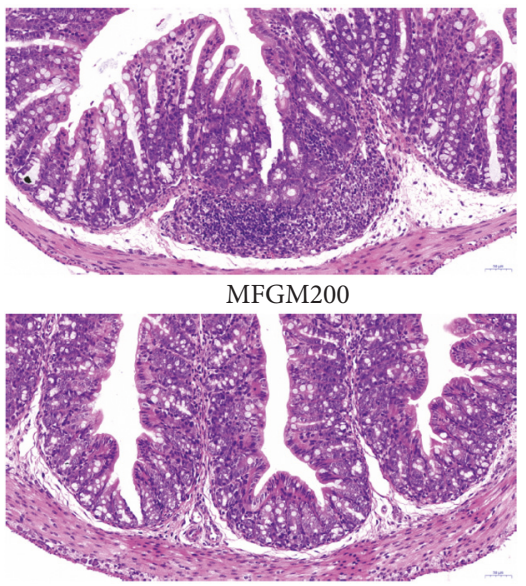

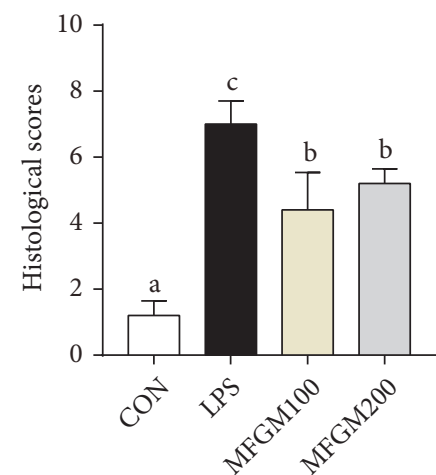

FIGURE 3: Effect of MFGM presupplementation from postnatal d 4 to $d 21$ on colon damage in LBW mice after LPS challenge. Mean values with their standard errors of the mean (SEM) ( $n=8$ pups/group). Within a row, means without a common letter $(a, b$, and $c)$ differ $(p<0.05)$.

To the best of our knowledge, this is the first to report that MFGM supplementation affects the epithelium integrity and toll-like receptors pathway in LBW neonatal mice.

First, MFGM supplementation from PND4 to PND21 improved the weight gain and growth performance in LBW pups. Studies showed that milk contains various bioactive compounds for infants, playing vital roles in regulating GIT development and protecting against infections during the early life of infants [29]. Milk fat globule membrane is a bioactive molecule better for gut health [13]. MFGM supplementation could increase the villus lengths and decrease crypt depths in the neonatal period of mice and, therefore, usually increase the utilization of nutrients [30, 31]. In this study, our results showed that MFGM supplementation (100 $\mathrm{mg} / \mathrm{kg} \mathrm{BW}$ or $200 \mathrm{mg} / \mathrm{kg} \mathrm{BW}$ ) could improve the growth performance of LBW pups from PND16 to PND21, which is consistent with the previous study [22]. The growthpromoting effects during this time zone could be explained by the increased needs of the neonates for growth but gradually decreased amount of secreted breast milk from PND14 [8, 32]. Consequently, further studies need to be done to elucidate how MFGM supplementation affects the growth performance at different ages.

Second, MFGM presupplementation improved the intestinal structure and barrier function and alleviated the intestinal inflammation after LPS challenge. In link with previous studies [33], intestinal villus was damaged by LPS challenge, suggesting that LPS could cause obstruction of intestinal development and histological damage during early life. As mentioned before, the GIT was important for postnatal nutrient acquisition [26]; damage of the intestinal morphology and structure was associated with 


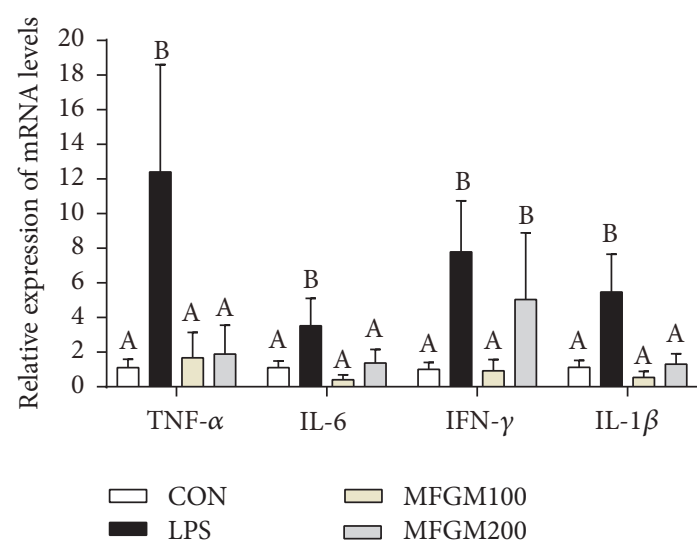

(a)

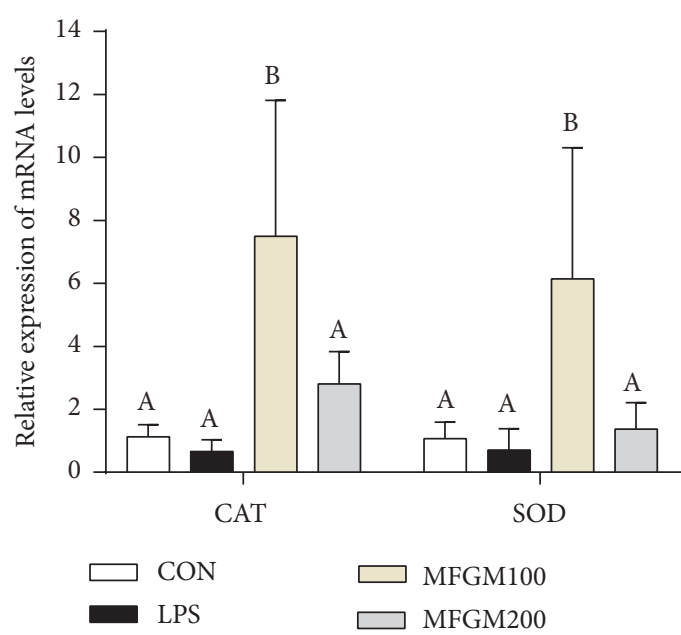

(c)

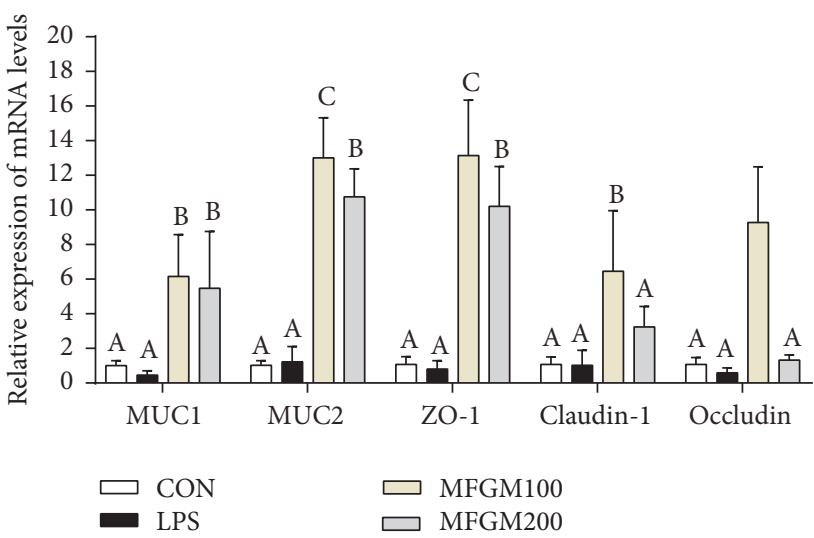

(b)

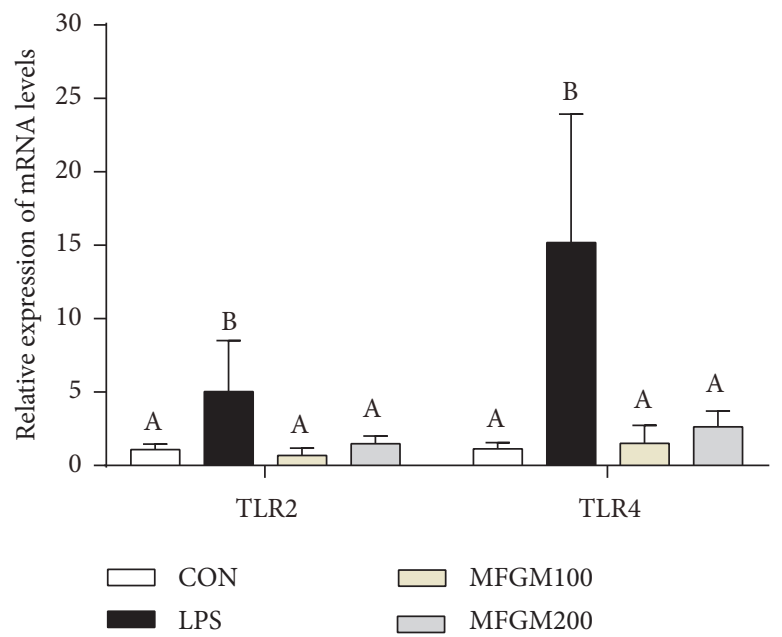

(d)

FIGURE 4: Effect of MFGM presupplementation from postnatal 44 to $d 21$ on the $m R N A$ abundance of genes in the ileum of LBW mice after LPS challenge. Mean values with their standard errors of the mean (SEM) ( $\mathrm{n}=8$ pups/group). Within a row, means without a common letter (A, $B$, and C) differ $(p<0.05)$.

disorder of digestion and absorption. In the current study, our results showed that MFGM presupplementation from PND 4 to PND 21 mitigated the intestinal histological damage and decreased the infiltration of intestinal lamina propria in ileum and colon of LBW mice challenged by LPS, which were in accordance with study carried out by Snow et al. [16]. Previous studies have also found that the intestinal epithelium forms the most important barrier among the internal and external environments [34]. The barrier is maintained by the tight junctions, including ZO-1, occludin, and claudin-1 [8, 33, 35]. Meanwhile, mucins (MUCs), the major components of mucous and a kind of glycoproteins, act as various roles in homeostasis [36] and can protect and lubricate the epithelial mucosa [37]. Our results showed that MFGM presupplementation increased the mRNA levels of ZO-1, claudin-1, and occludin in ileum and colon of the LPS-induced LBW mice and improved the expression of MUC1 and MUC2, in accordance with the study carried out by Snow et al. [16]. These results suggested that MFGM supplementation improves the development of villi morphology, as well as maintaining the intestinal integrity and barrier function by improving the expression levels of tight junction and MUCs.

Third, MFGM presupplementation improved the intestinal oxidative stress during LPS challenge. Due to the suddenly increased oxygen concentration at birth, a large number of oxygen free radicals were produced in the intestine $[8$, 38-40], ultimately leading to oxidative stress in the GIT of the neonates $[38,41]$. According to previous studies [8, $10,28,38,42]$, the impaired GIT of IUGR neonates is prone to produce a large amount of oxygen free radicals, thereby exacerbating their oxidative stress. The intestinal morphology and barrier of neonatal mice were disturbed by LPS, leading to increased intestinal permeability and damaged tight junction, ultimately resulting in oxidative stress [43-45]. Oxidative stress is frequently associated with intestinal epithelial barrier and inflammatory cytokines, such as tight junction and proinflammatory cytokines, respectively $[8,46]$. Previous studies showed that oxidative stress could increase the proinflammatory cytokine (TNF- $\alpha$ ) expression and decreased the tight junction mRNA level in the intestinal mucosa of piglets $[16,46,47]$, where oxidative stress could be 


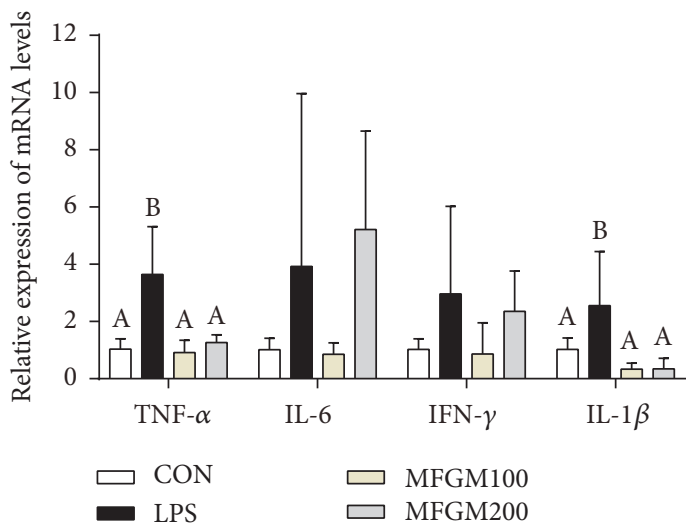

(a)

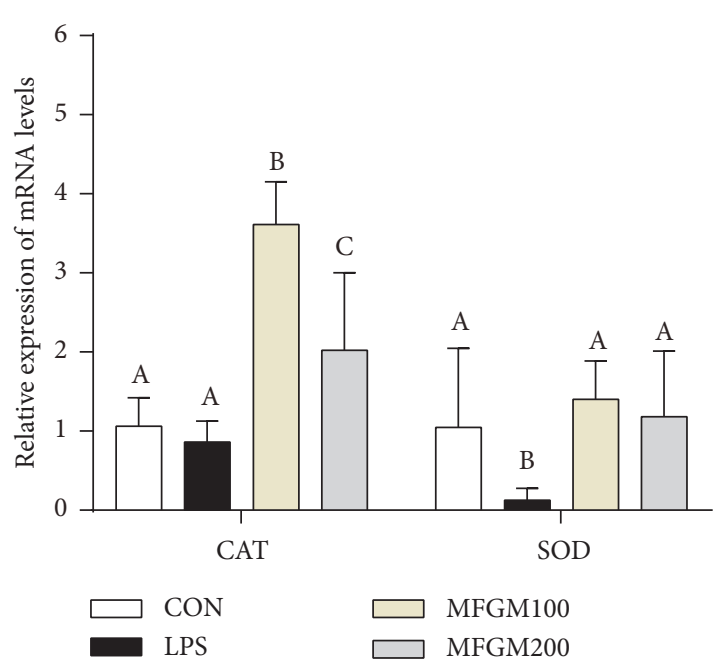

(c)

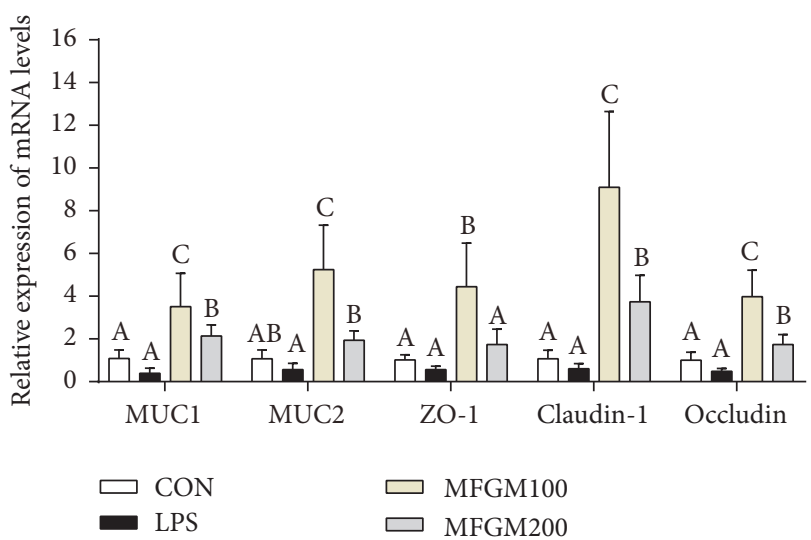

(b)

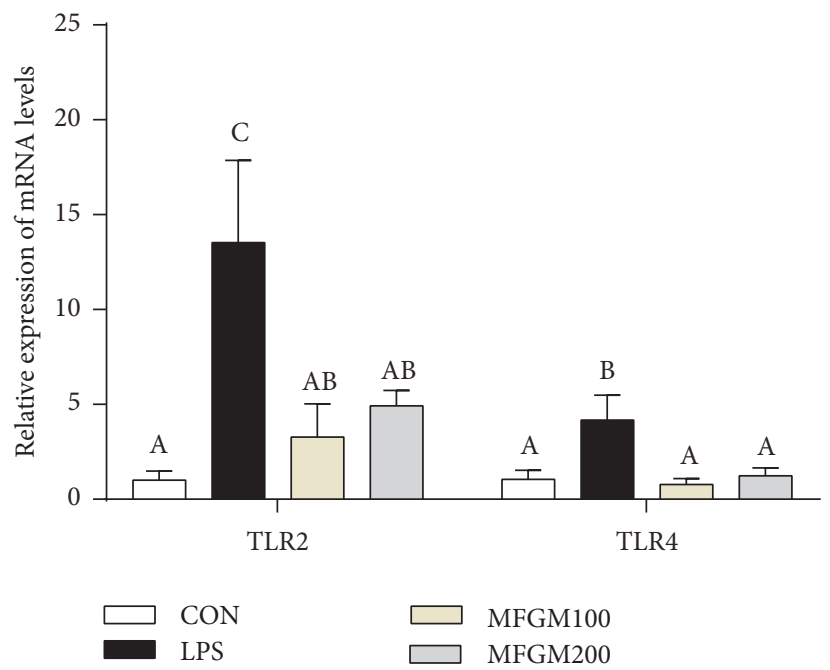

(d)

FIGURE 5: Effect of MFGM presupplementation from postnatal $d 4$ to $d 21$ on the $m R N A$ abundance of genes in the colon of LBW mice after LPS challenge. Mean values with their standard errors of the mean (SEM) ( $\mathrm{n}=8$ pups/group). Within a row, means without a common letter (A, $\mathrm{B}$, and $\mathrm{C})$ differ $(p<0.05)$.

a vital factor leading to intestinal dysfunction in LBW piglets [8]. To investigate the oxidative stress in LBW pups after LPS challenge, the redox status of plasma and intestine was determined. In the current study, the plasma concentration of SOD was increased by MFGM presupplementation from PND4 to PND21 in LPS-induced LBW mice. Meanwhile, MFGM presupplementation increased the expression levels of SOD and CAT in both ileum and colon after LPS challenge. It was well known that SOD and CAT are antioxidant enzyme systems to maintain cellular integrity and tissue redox homeostasis $[48,49]$. And MDA is the most important biomarker of lipid peroxidation products and may impair intestinal integrity and intestinal permeability [8]. Thus, MFGM supplementation could ameliorate intestinal dysfunction in LBW pups by enhancing the antioxidant systems.

Fourth, mammalian toll-like receptors (TLRs) play a vital role in signal transduction during pathogen invasion, inflammatory, and immune response [50,51]. Previous studies revealed that TLR2 played important role in inflammatory response [50,51]. Meanwhile, it was well known that intestinal epithelium cells recognize LPS through the membrane protein of TLR4. LPS can cause intestinal inflammatory and promote the expression levels of proinflammatory cytokines via activation of the TLR4/NF- $\kappa \mathrm{B}$ signaling pathway [52]. According to our results, the expression levels of TLR4 and TLR2 were increased in the ileum and colon of LPS-challenged LBW mice, while being significantly abolished by MFGM presupplementation. Meanwhile, the genes expression of proinflammatory cytokines was decreased in the MFGM presupplementation group. In summary, our results showed that MFGM presupplementation decreased the expression level of proinflammatory cytokines by modulating TLR signaling pathway in LPS-induced LBW mice, which was consistent with previous studies $[16,33]$.

In conclusion, using the LPS-induced neonatal LBW mice model, we demonstrated that MFGM supplementation improved the growth performance of the neonatal mice during their early life. MFGM presupplementation could also alleviate the intestinal damage induced by LPS challenge in LBW mice through increasing the mRNA levels of tight 
junction, intestinal mucosal barrier, and antioxidant enzyme, while reducing the expression of proinflammatory cytokines by inhibiting TLR2 and TLR4 signaling. This study disclosed that MFGM is a functional nutrient with an ability to improve the growth performance of LBW mouse pups, especially during the LPS challenge, and provides a new approach for treatment or prevention of intestinal inflammatory in LBW neonates during their early life.

$\begin{array}{ll}\text { Abbreviations } \\ \text { LBW: } & \text { Low birth weight } \\ \text { PND: } & \text { Postnatal day } \\ \text { LPS: } & \text { Lipopolysaccharides } \\ \text { ANOVA: } & \text { One-way analysis of variance } \\ \text { IL-1 } \beta \text { : } & \text { Interleukin-1 beta } \\ \text { IL-6: } & \text { Interleukin-6 } \\ \text { TNF- } \alpha: & \text { Tumor necrosis factor alpha } \\ \text { IFN- } \gamma: & \text { Interferon-gamma } \\ \text { SD: } & \text { Standard deviation } \\ \text { CAT: } & \text { Catalase } \\ \text { SOD: } & \text { Superoxide dismutase } \\ \text { TLRs: } & \text { Toll-like receptors } \\ \text { TLR2: } & \text { Toll-like receptor } 2 \\ \text { TLR4: } & \text { Toll-like receptor } 4 \\ \text { MUC1: } & \text { Mucin } 1 \\ \text { MUC2: } & \text { Mucin } 2 .\end{array}$

\section{Data Availability}

The data used to support the findings of this study are available from the corresponding author (jkywjj@hotmail.com) upon request.

\section{Conflicts of Interest}

The authors declare that the research was conducted in the absence of any commercial or financial relationships that could be construed as potential conflicts of interest.

\section{Authors' Contributions}

Junjun Wang, Shimeng Huang, Dandan Han, and Cuiping Feng designed the experiments. Shimeng Huang and Zhenhua $\mathrm{Wu}$ conducted the experiment. Shimeng Huang and Zhenhua Wu carried out the experiments and collected the samples. Shimeng Huang, Zhenhua Wu, and Cong Liu performed the analysis of samples. Shimeng Huang, Cong Liu, Dandan Han, Shilan Wang, and Zhenhua Wu analyzed the data. Shimeng Huang, Cong Liu, and Junjun Wang wrote the manuscript. All authors read and approved the final manuscript.

\section{Acknowledgments}

The MFGM was provided by the Sanyuan Foods Co. Ltd. in Beijing, China. We thank the experimental animal platform of China Agricultural University in Beijing, China, for the assistances in this study. This work was supported by the Beijing Municipal Natural Science Foundation (S170001), the
National Natural Science Foundation of China (31630074), the National Key Research and Development Program of China (2016YFD0500506 and 2018YDF0501002), the 111 Project (B16044), and the Jinxinnong Animal Science Developmental Foundation.

\section{Supplementary Materials}

Supplementary Table S1: the information of primer sequences used for qRT-PCR quantification of related genes. (Supplementary Materials)

\section{References}

[1] M. A. Selak, B. T. Storey, I. Peterside, and R. A. Simmons, "Impaired oxidative phosphorylation in skeletal muscle of intrauterine growth-retarded rats," American Journal of Physiology-Endocrinology and Metabolism, vol. 285, no. 1, pp. E130E137, 2003.

[2] G. Wu, F. W. Bazer, J. M. Wallace, and T. E. Spencer, "Boardinvited review: Intrauterine growth retardation: Implications for the animal sciences," Journal of Animal Science, vol. 84, no. 9, pp. 2316-2337, 2006.

[3] X. Wang, Y. Zhu, C. Feng et al., "Innate differences and colostrum-induced alterations of jejunal mucosal proteins in piglets with intra-uterine growth restriction," British Journal of Nutrition, vol. 119, no. 7, pp. 734-747, 2018.

[4] G. Su, M. S. Lund, and D. Sorensen, "Selection for litter size at day five to improve litter size at weaning and piglet survival rate," Journal of Animal Science, vol. 85, no. 6, pp. 1385-1392, 2007.

[5] H. Aly, J. Davies, M. El-Dib, and A. Massaro, "Renal function is impaired in small for gestational age premature infants," The Journal of Maternal-Fetal and Neonatal Medicine, vol. 26, no. 4, pp. 388-391, 2013.

[6] A. Tzschoppe, E. Struwe, W. Rascher et al., "Intrauterine growth restriction (IUGR) is associated with increased leptin synthesis and binding capability in neonates," Clinical Endocrinology, vol. 74, no. 4, pp. 459-466, 2011.

[7] L. Hu, Y. Liu, C. Yan et al., "Postnatal nutritional restriction affects growth and immune function of piglets with intrauterine growth restriction," British Journal of Nutrition, vol. 114, no. 1, pp. 53-62, 2015.

[8] P. Zheng, Y. Song, Y. Tian et al., "Dietary arginine supplementation affects intestinal function by enhancing antioxidant capacity of a nitric oxide-independent pathway in low-birthweight piglets," Journal of Nutrition, vol. 148, no. 11, pp. 17511759, 2018.

[9] S. E. Pinney and R. A. Simmons, "Metabolic programming, Epigenetics, and gestational diabetes mellitus," Current Diabetes Reports, vol. 12, no. 1, pp. 67-74, 2012.

[10] N. Li, W. Wang, G. Wu, and J. Wang, "Nutritional support for low birth weight infants: Insights from animal studies," British Journal of Nutrition, vol. 117, no. 10, pp. 1390-1402, 2017.

[11] P. D. Gluckman, M. A. Hanson, C. Cooper, and K. L. Thornburg, "Effect of in utero and early-life conditions on adult health and disease," The New England Journal of Medicine, vol. 359, no. 1, pp. 61-73, 2008.

[12] X. Zou, Z. Guo, Q. Jin et al., "Composition and microstructure of colostrum and mature bovine milk fat globule membrane," Food Chemistry, vol. 185, pp. 362-370, 2015. 
[13] C. Bourlieu and M.-C. Michalski, "Structure-function relationship of the milk fat globule," Current Opinion in Clinical Nutrition \& Metabolic Care, vol. 18, no. 2, pp. 118-127, 2015.

[14] C. Bourlieu, K. Bouzerzour, S. Ferret-Bernard et al., "Infant formula interface and fat source impact on neonatal digestion and gut microbiota," European Journal of Lipid Science and Technology, vol. 117, no. 10, pp. 1500-1512, 2015.

[15] R. C. Sprong, M. F. E. Hulstein, T. T. Lambers, and R. Van Der Meer, "Sweet buttermilk intake reduces colonisation and translocation of Listeria monocytogenes in rats by inhibiting mucosal pathogen adherence," British Journal of Nutrition, vol. 108, no. 11, pp. 2026-2033, 2012.

[16] D. R. Snow, R. E. Ward, A. Olsen, R. Jimenez-Flores, and K. J. Hintze, "Membrane-rich milk fat diet provides protection against gastrointestinal leakiness in mice treated with lipopolysaccharide," Journal of Dairy Science, vol. 94, no. 5, pp. 22012212, 2011.

[17] T. Li, J. Gao, M. Du, J. Song, and X. Mao, "Milk fat globule membrane attenuates high-fat diet-induced obesity by inhibiting adipogenesis and increasing uncoupling protein 1 expression in white adipose tissue of mice," Nutrients, vol. 10, no. 3, pp. 331342, 2018.

[18] A. T. Mudd, L. S. Alexander, K. Berding et al., "Dietary prebiotics, milk fat globule membrane, and lactoferrin affects structural neurodevelopment in the young piglet," Frontiers in Pediatrics, vol. 4, pp. 1-10, 2016.

[19] N. Timby, E. Domellöf, O. Hernell, B. Lönnerdal, and M. Domellöf, "Neurodevelopment, nutrition, and growth until 12 mo of age in infants fed a low-energy, low-protein formula supplemented with bovine milk fat globule membranes: a randomized controlled trial," American Journal of Clinical Nutrition, vol. 99, no. 4, pp. 860-868, 2014.

[20] O. Hernell, N. Timby, M. Domellöf, and B. Lönnerdal, "Clinical benefits of milk fat globule membranes for infants and children," Journal of Pediatrics, vol. 173, pp. S60-S65, 2016.

[21] G. Bhinder, J. M. Allaire, C. Garcia et al., "Milk fat globule membrane supplementation in formula modulates the neonatal gut microbiome and normalizes intestinal development," Scientific Reports, vol. 7, pp. 1-15, 2017.

[22] L. R. Brink and B. Lönnerdal, "The role of milk fat globule membranes in behavior and cognitive function using a suckling rat pup supplementation model," The Journal of Nutritional Biochemistry, vol. 58, pp. 131-137, 2018.

[23] G. Lin, C. Liu, C. Feng et al., "Metabolomic analysis reveals differences in umbilical vein plasma metabolites between normal and growth-restricted fetal pigs during late gestation," Journal of Nutrition, vol. 142, no. 6, pp. 990-998, 2012.

[24] Y. Ji, Z. Dai, S. Sun et al., "Hydroxyproline attenuates dextran sulfate sodium-induced colitis in mice: involvment of the NF$\kappa \mathrm{B}$ signaling and oxidative stress," Molecular Nutrition \& Food Research, vol. 62, no. 21, Article ID e1800494, 2018.

[25] Y. Nishiyama, T. Kataoka, K. Yamato, T. Taguchi, and K. Yamaoka, "Suppression of dextran sulfate sodium-induced colitis in mice by radon inhalation," Mediators of Inflammation, vol. 2012, Article ID 239617, 11 pages, 2012.

[26] R. D’Inca, C. Gras-Le Guen, L. Che, P. T. Sangild, and I. Le Huërou-Luron, "Intrauterine growth restriction delays feedinginduced gut adaptation in term newborn pigs," Neonatology, vol. 99, no. 3, pp. 208-216, 2011.

[27] J. Wang, L. Chen, D. Li et al., "Intrauterine growth restriction affects the proteomes of the small intestine, liver, and skeletal muscle in newborn pigs," Journal of Nutrition, vol. 138, no. 1, pp. 60-66, 2008.

[28] J. Wang, Z. Wu, D. Li et al., "Nutrition, epigenetics, and metabolic syndrome," Antioxidants \& Redox Signaling, vol. 17, no. 2, pp. 282-301, 2012.

[29] W. Z. Wu, X. Q. Wang, G. Y. Wu, S. W. Kim, F. Chen, and J. J. Wang, "Differential composition of proteomes in sow colostrum and milk from anterior and posterior mammary glands," Journal of Animal Science, vol. 88, no. 8, pp. 2657-2664, 2010.

[30] K.-Y. Yeh and M. Yeh, "Use of pup in a cup model to study gastrointestinal development: Interaction of nutrition and pituitary hormones," Journal of Nutrition, vol. 123, no. 2, pp. 378-381, 1993.

[31] I. C. Teller, H. Hoyer-Kuhn, H. Brönneke et al., "Complex lipid globules in early-life nutrition improve long-term metabolic phenotype in intra-uterine growth-restricted rats," British Journal of Nutrition, vol. 120, no. 7, pp. 763-776, 2018.

[32] Y. Chen, D. Mou, L. Hu et al., "Effects of maternal low-energy diet during gestation on intestinal morphology, disaccharidase activity, and immune response to lipopolysaccharide challenge in pig offspring," Nutrients, vol. 9, no. 10, 2017.

[33] H. Zhu, H. Wang, S. Wang et al., "Flaxseed oil attenuates intestinal damage and inflammation by regulating necroptosis and TLR4/NOD signaling pathways following lipopolysaccharide challenge in a piglet model," Molecular Nutrition \& Food Research, vol. 62, no. 9, Article ID e1700814, 2018.

[34] K. R. Groschwitz and S. P. Hogan, "Intestinal barrier function: molecular regulation and disease pathogenesis," The Journal of Allergy and Clinical Immunology, vol. 124, no. 1, pp. 3-20, 2009.

[35] E. Dejana, "Endothelial cell-cell junctions: happy together," Nature Reviews Molecular Cell Biology, vol. 5, no. 4, pp. 261-270, 2004.

[36] E. Levi, D. S. Klimstra, N. V. Adsay, A. Andea, and O. Basturk, "MUC1 and MUC2 in pancreatic neoplasia," Journal of Clinical Pathology, vol. 57, no. 5, pp. 456-462, 2004.

[37] A. P. Corfield, D. Carroll, N. Myerscough, and C. S. Probert, "Mucins in the gastrointestinal tract in health and disease," Frontiers in Bioscience: A Journal and Virtual Library, vol. 6, pp. D1321-1357, 2001.

[38] W. Wang, J. Degroote, C. Van Ginneken et al., "Intrauterine growth restriction in neonatal piglets affects small intestinal mucosal permeability and mRNA expression of redox-sensitive genes," The FASEB Journal, vol. 30, no. 2, pp. 863-873, 2016.

[39] J. Yin, W. Ren, G. Liu et al., "Birth oxidative stress and the development of an antioxidant system in newborn piglets," Free Radical Research, vol. 47, no. 12, pp. 1027-1035, 2013.

[40] Y. Surh, J. K. Kundu, H. Na, and J. Lee, "Redox-sensitive transcription factors as prime targets for chemoprevention with anti-inflammatory and antioxidative phytochemicals," Journal of Nutrition, vol. 135, no. 12, pp. 2993S-3001S, 2005.

[41] J. K. Friel, R. W. Friesen, S. V. Harding, and L. J. Roberts, "Evidence of oxidative stress in full-term healthy infants," Pediatric Research, vol. 56, no. 6, pp. 878-882, 2004.

[42] X. Wang, G. Lin, C. Liu et al., “Temporal proteomic analysis reveals defects in small-intestinal development of porcine fetuses with intrauterine growth restriction," The Journal of Nutritional Biochemistry, vol. 25, no. 7, pp. 785-795, 2014.

[43] M. Mittal, M. R. Siddiqui, K. Tran, S. P. Reddy, and A. B. Malik, "Reactive oxygen species in inflammation and tissue injury," Antioxidants \& Redox Signaling, vol. 20, no. 7, pp. 1126-1167, 2014. 
[44] N. R. P. G. Kallapura, X. H. Velasco, B. Hargisand, and G. Tellez, "Mechanisms involved in lipopolysaccharide derived ROS and RNS oxidative stress and septic shock," Journal of Microbiology Research and Reviews, vol. 2, pp. 6-11, 2014.

[45] P. Libby, "Inflammatory mechanisms: the molecular basis of inflammation and disease," Nutrition Reviews, vol. 65, no. 3, pp. S140-S146, 2007.

[46] B. Rada, P. Gardina, T. G. Myers, and T. L. Leto, "Reactive oxygen species mediate inflammatory cytokine release and EGFRdependent mucin secretion in airway epithelial cells exposed to Pseudomonas pyocyanin," Mucosal Immunology, vol. 4, no. 2, pp. 158-171, 2011.

[47] G. Buonocore, F. Bazzini, F. Proietti et al., "The free radical diseases of newborn," Journal of Pediatric Biochemistry, vol. 6, no. 2, pp. 73-78, 2016.

[48] Y. Ozsurekci and K. Aykac, "Oxidative Stress Related Diseases in Newborns," Oxidative Medicine and Cellular Longevity, vol. 2016, 2016.

[49] L. He, T. He, S. Farrar, L. Ji, T. Liu, and X. Ma, "Antioxidants maintain cellular redox homeostasis by elimination of reactive oxygen species," Cellular Physiology and Biochemistry, vol. 44, no. 2, pp. 532-553, 2017.

[50] E.-K. Jo, "Mycobacterial interaction with innate receptors: TLRs, C-type lectins, and NLRs," Current Opinion in Infectious Diseases, vol. 21, no. 3, pp. 279-286, 2008.

[51] S. Janssens and R. Beyaert, "Role of Toll-like receptors in pathogen recognition," Clinical Microbiology Reviews, vol. 16, no. 4, pp. 637-646, 2003.

[52] T. Liu, L. Zhang, D. Joo, and S. Sun, "NF- $\kappa$ B signaling in inflammation," Signal Transduction and Targeted Therapy, vol. 2, pp. 1-9, 2017. 


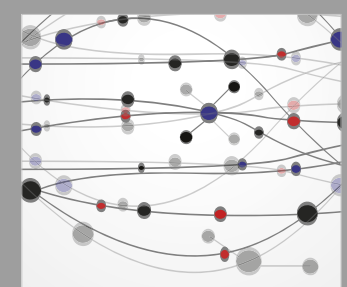

The Scientific World Journal
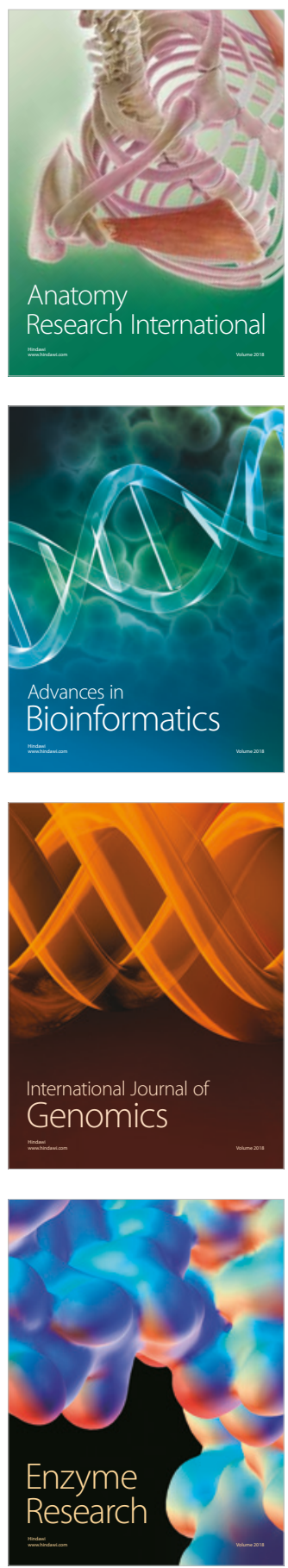
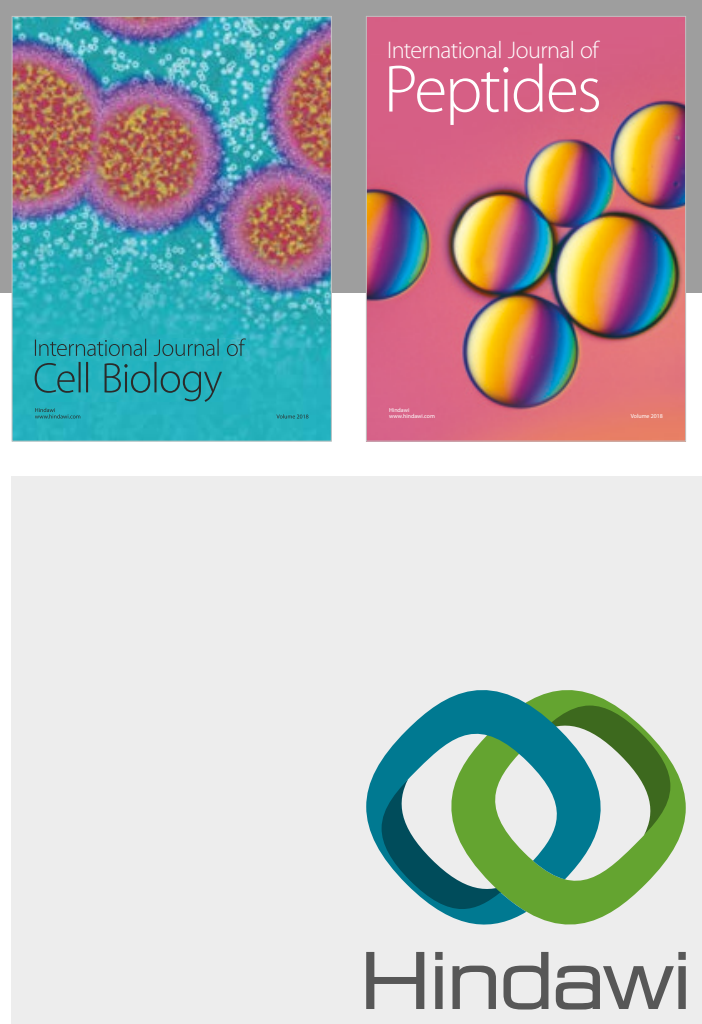

Submit your manuscripts at

www.hindawi.com
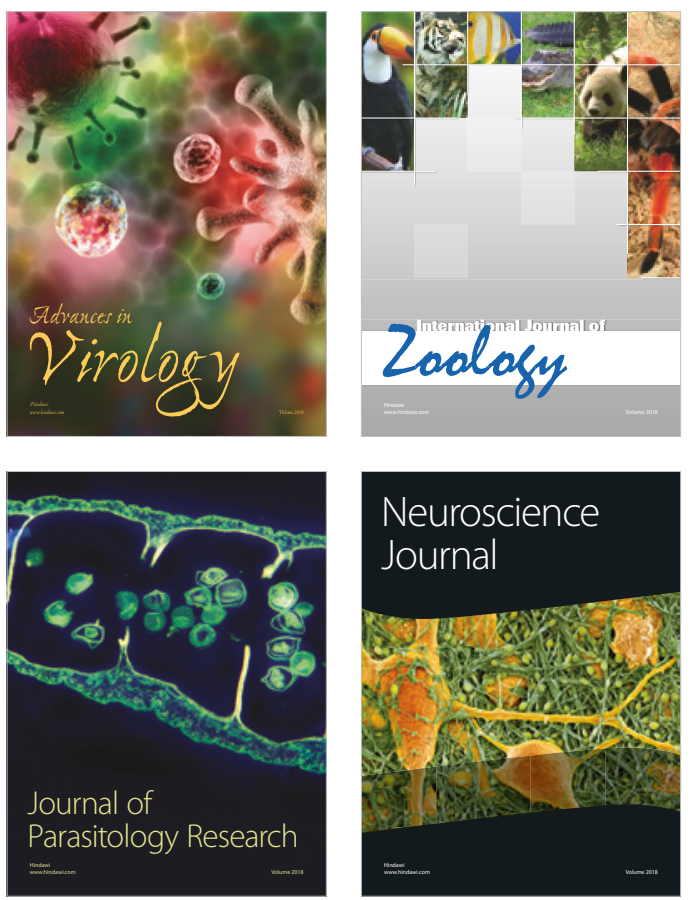
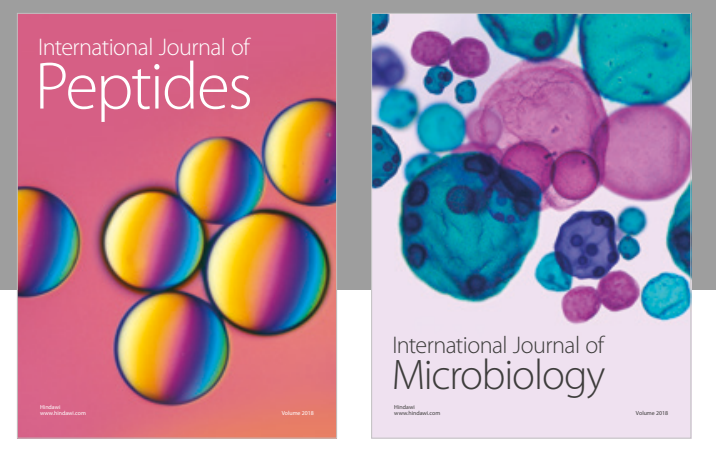

nternational Journal of Microbiology
Journal of
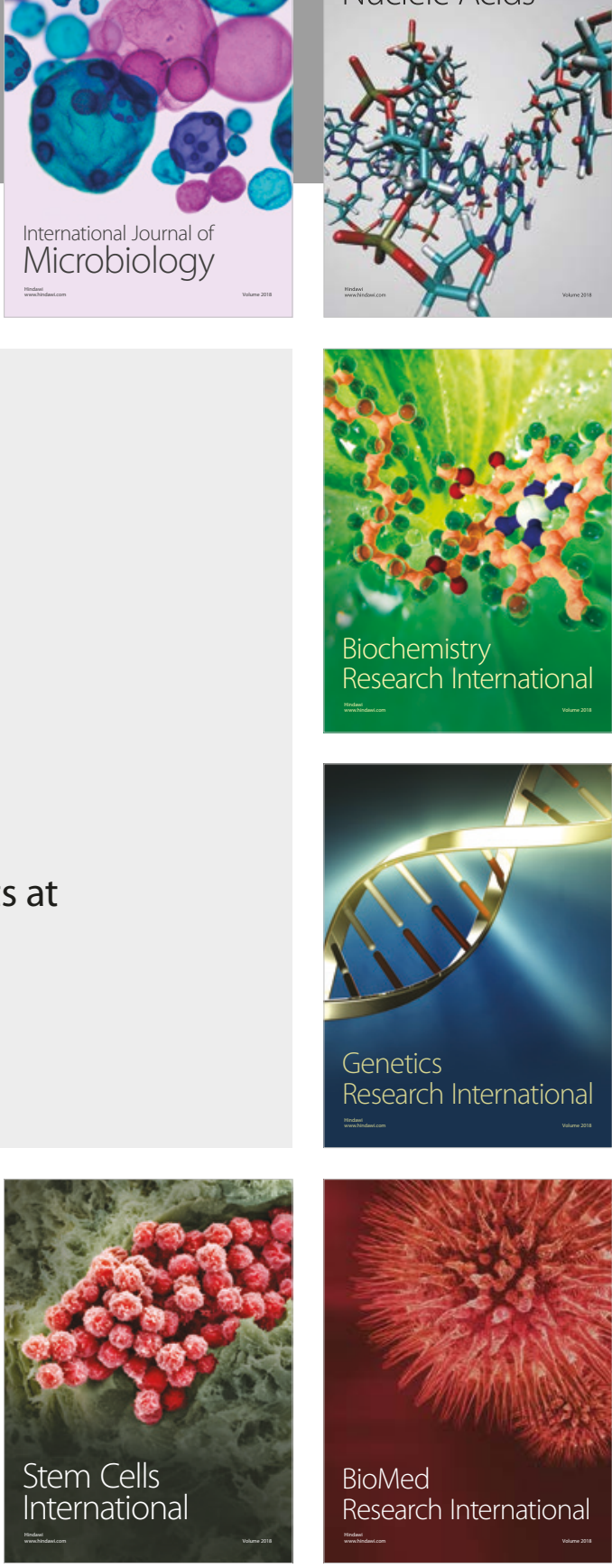
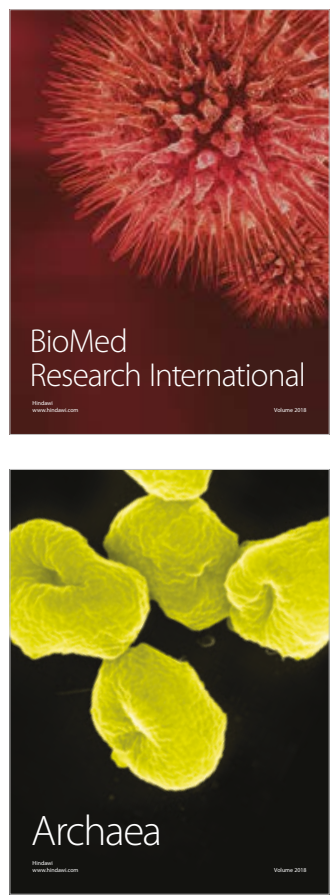